\title{
Nutrition et métabolisme cuprique de la vache
}

\author{
par \\ Jean-Luc ILARI \\ Docteur-ingénieur \\ Professeur de Génie alimentaire \\ E.N.I.T.I.A.A. "La Géraudière " - 44000 Nantes
}

Après la mise en évidence définitive par Davis [1] en 1931, du rôle néfaste du cuivre dans le lait et les produits laitiers, où il est en particulier responsable des goûts dits " métalliques » ou d'oxydation, la majeure partie des travaux en recherche et en technologie a conclu au bannissement de ce métal de l'industrie laitière. Pourtant le cuivre existe à l'état naturel chez l'être vivant, constitue un oligoélément indispensable à une alimentation équilibrée, est particulièrement nécessaire au jeune en croissance, et ses cas de carence et de toxicité chez l'animal, quoique connus des zootechniciens, sont relativement restés dans l'ombre.

Il nous apparaît donc intéressant, après avoir tenté de réhabiliter le cuivre en technologie des pâtes pressées, d'essayer d'éclairer dans l'espèce bovine, le problème de la nutrition et du métabolisme cuprique. Cette revue non exhaustive et très sommaire des sources alimentaires, du seuil de toxicité, et des variations pathologiques ou accidentelles du cuivre chez la vache devrait permettre, outre un regain d'intérêt, de rapprocher un certain nombre de données utiles au professionnel, éleveur ou laitier. Quoique situées en amont des problèmes posés par le cuivre dans l'industrie laitière, nous pensons que ces quelques idées contribueront à les éclairer d'une façon différente, donc peut-être à aider à les résoudre.

\section{I. - LES APPORTS ALIMENTAIRES DE CUIVRE}

C'est à Lucie RANDOIN [2] et à son impulsion déterminante que l'on doit en France l'établissement de tables de composition chimique d'aliments, et la réalisation des nombreuses analyses qui permirent de dresser ces tables. Ses premières préoccupations, d'origine nutritionnelle, s'appliquèrent en particulier aux aliments de l'homme. Mais les travaux analytiques se développèrent rapidement sur les 
aliments du bétail, qu'ils soient traditionnels ou de conception plus récente. Les zootechniciens souhaitaient, avec l'aide de l'analyse chimique, arriver à définir et éventuellement faire évoluer les transformations biochimiques dans l'appareil digestif de la vache de façon à obtenir un produit final (en l'occurence, le lait ou la viande) soit en quantité supérieure, soit de qualité meilleure.

Ainsi, pendant longtemps s'est-on surtout attaché au dosage du cuivre global, comme le permettaient les méthodes qui s'appliquaient avec plus ou moins de précision sur tel ou tel matériau biologique (organes en particulier). Seules les notions physico-chimiques modernes de chimie atomique ont permis de mettre en évidence et de mieux comprendre les diverses formes chimiques que pouvait prendre l'élément cuivre, ainsi que leur importance biochimique relative. Néanmoins, si de nombreux dosages chimiques du cuivre ont été effectués dans les aliments en vue d'étudier de nombreux cas de toxicité chronique, ou de carence (surtout chez l'animal jeune) la majorité des résultats que nous possédons néglige l'aspect qualitatif au profit du quantitatif.

Il faut étudier comparativement les divers aliments du bétail pour comprendre qu'il existe des sources plus ou moins riches en cuivre. De plus la forme sous laquelle le cuivre est apporté par l'aliment, organique ou minérale, peut grandement influencer son utilisation biologique.

Si les aliments du bétail «traditionnels » paraissent moyennement riches en cuivre, puisque les teneurs varient, d'après le Commonwealth Bureau of Animal Nutrition [3] de 3 à $5 \mathrm{mg}$ par $\mathrm{kg}$ ou p.p.m., les techniques d'alimentation moderne utilisent des aliments relativement plus pauvres, tels que le riz, les farines, les racines et les tubercules, et d'autres relativement plus riches tels que les abats, les farines d'os ou de poisson, les tourteaux, etc.

Même si l'utilisation digestive en reste toujours assez floue, nous connaissons les teneurs en cuivre de tous les aliments du bétail tels qu'ils ont été donnés par Jaulmes P. et Hamelle G. [4].

Tableau des teneurs en cuivre de quelques aliments pour bovins exprimées en p.p.m. ( $\mathrm{mg}$ par $\mathrm{kg}$ )

Farine de blé (matière sèche)

Blé (matière sèche)

Riz (matière sèche)

Seigle (matière sèche)

Avoine (matière sèche)

Maïs (en grains)

Choux (état frais)

Foin de luzerne 
Foin de trèfle

Luzerne fraîche

Trèfle frais

Paille d'avoine

Tourteaux d'arachide

Tourteaux de soja

Tourteaux de palmiste

Farine de poisson (état sec)

Levure de bière (état $\mathrm{sec}$ )

4
1
1
4,5
23
12
27
9 à 22
20

Les aliments qui apportent le plus de cuivre sont donc les aliments concentrés comme les tourteaux ou les farines de poisson, mais leur emploi reste limité puisqu'ils ne constituent pas l'aliment de base et n'entrent qu'en complément dans les rations. L'ensilage se situe au même niveau que le foin.

Dans le cas d'aliments plus élaborés, tels que les granulés et les comprimés, une étude a été réalisée aux Etats-Unis par Heckman [5] sur cinq types bien définis d'aliments pour bétail préparés suivant des formules équilibrées, aliments se présentant sous la forme de concentrés secs. Nous donnons ci-dessous le tableau des résultats obtenus. Ces résultats sont dignes de confiance car ils ont donné lieu à une étude statistique réalisée sur plusieurs laboratoires différents. Les dosages effectués visaient à mettre au point la méthode officielle de dosage du cuivre A.O.A.C.

Tableau des teneurs en cuivre

Type d'aliment concentré

$\mathrm{a}$
$\mathrm{b}$
$\mathrm{c}$
$\mathrm{d}$
$\mathrm{e}$

Teneur en p.p.m.

10 à 25

50 à 100

20 à 35

10 à 25

15 à 30

Ce tableau permet d'entrevoir une évolution dans les nutriments apportés aux bovins. Les aliments non seulement sont sous une forme plus concentrée (forme sèche), mais leurs teneurs paraissent aussi beaucoup plus élevées. On peut expliquer ce phénomène par un enrichissement volontaire en cuivre au cours du traitement technologique de préparation de l'aliment concentré, ainsi que par le simple fait de la concentration. De plus, cette nouvelle forme d'alimentation 
donne une importance plus grande à l'eau de boisson, dont trop souvent encore la composition minérale n'est pas vérifiée. Or les besoins en eau des bovins sont très élevés et, quoique proportionnels à la quantité de nourriture ingérée, ils dépendent de certains facteurs extérieurs comme la température. Nous pouvons les résumer dans le tableau suivant.

Besoins des bovins en eau

\begin{tabular}{|c|c|c|}
\hline Catégories d'animaux & $\begin{array}{l}\text { Température } \\
\text { environnante }\end{array}$ & $\begin{array}{l}\text { Eau nécessaire par } 1 / \mathrm{kg} \\
\text { matière sèche consommée }\end{array}$ \\
\hline Bovins supérieurs à $100 \mathrm{~kg}$ & $\begin{array}{l}-17^{\circ} \mathrm{C} \text { à }+10^{\circ} \mathrm{C} \\
10^{\circ} \mathrm{C} \text { à } 15^{\circ} \mathrm{C} \\
15^{\circ} \mathrm{C} \text { à } 21^{\circ} \mathrm{C} \\
21^{\circ} \mathrm{C} \text { à } 27^{\circ} \mathrm{C} \\
\text { au delà de } 27^{\circ} \mathrm{C}\end{array}$ & $\begin{array}{l}3,5 \\
3,6 \\
4,1 \\
4,7 \\
5,5\end{array}$ \\
\hline Vaches en lactation & \multicolumn{2}{|c|}{ Ajouter $0,87 \mathrm{~kg}$ d'eau par litre de lait produit. } \\
\hline Vaches en gestation. & \multicolumn{2}{|c|}{$\begin{array}{l}\text { Pendant les quatre derniers mois, multiplier les } \\
\text { valeurs ci-dessus par le facteur } 1,5 \text {. }\end{array}$} \\
\hline
\end{tabular}

Ces chiffres sont ceux fournis par l'Agricultural Research Council [6] et ils mettent en évidence l'importance que pourrait avoir une eau plus ou moins enrichie en cuivre. Or l'enrichissement par contamination est toujours possible, par l'intermédiaire de facteurs comme les cuves, les tuyauteries et les robinets en cuivre ou en alliages. Ainsi Golse [7] rapportait que les eaux passant par des conduites en cuivre peuvent s'enrichir jusqu'à plus de cent fois en ce métal. Nous avons nous-même trouvé [8] dans des résidus de bi-distillation (eau distillée qui persiste au fond du ballon de bi-distillation) des concentrations allant jusqu'à 75 p.p.m., ce qui dénote une présence de cuivre certaine dans les eaux distillées.

Aussi convient-il de garder à l'esprit les risques éventuels de toxicité lorsque le bétail est nourri essentiellement avec les aliments " concentrés " et par suite consomme beaucoup plus d'eau de boisson. Et il ne faut pas oublier, d'une façon beaucoup plus générale, que l'eau peut intervenir par sa "composition » sur l'équilibre nutritionnel des animaux (avec ou sans intervention du cuivre). 


\section{II. - LES BESOINS DES BOVINS EN CUIVRE}

Pour les bovins, comme pour d'autres animaux, ils sont difficiles à définir par suite des nombreuses interactions métaboliques qui peuvent avoir lieu, avec des éléments comme le cobalt, le molybdène, le fer, ou des composés chimiques comme les sulfates. De plus, si les carences, réalisées expérimentalement ou constatées, prouvent le caractère indispensable du cuivre, l'estimation biologique du facteur carence est très difficile à interpréter à cause de l'excrétion du type hépato-instestinale du métal lorsqu'il est injecté par voie intraveineuse à l'animal. Aussi les estimations des besoins restent-elles floues et sujettes à caution, d'autant plus que ce besoin paraît évoluer en fonction de l'âge de l'animal. Jacquot [9] cite des besoins de 50 à $70 \mathrm{mg}$ par jour pour des bovins adultes, et se réfère à Mitchell dans le cas de jeunes animaux, tels le veau, qui ont besoin au minimum de $9 \mathrm{mg}$ de cuivre par $\mathrm{kg}$ de matière sèche ingérée. Il apparaît que des concentrations de $10 \mathrm{mg} / \mathrm{kg}$ (10 p.p.m.) par rapport à la matière sèche, incompatibles avec des signes de carence, couvriraient donc les besoins, ce qui les fait recommander par l'Agicultural Research Council [10]. Si le chiffre de 10 p.p.m. paraît donc recommandable dans la majorité des cas, ces concentrations peuvent être largement augmentées lorsqu'il s'agit de cas de carence particuliers qu'il faut étudier et traiter individuellement pour satisfaire la couverture des besoins.

\section{III. - LE METABOLISME DU CUIVRE}

Nous allons, dans le cas de la vache, essayer de résumer le plus clairement possible les idées développées ces derniènes années dans ce domaine, en négligeant le problème des interrelations métaboliques entres les divers oligo-éléments, trop complexe pour notre propos, mais que la science vétérinaire ne peut se permettre d'ignorer.

\section{Absorption}

Le cuivre alimentaire est surtout absorbé dans la partie médiane de l'intestin grêle, et sa digestibilité chez les ruminants serait d'après Comar, Davis et Singer [11] de l'ordre de 28 p. 100. La formation éventuelle dans la lumière intestinale de sulfure de cuivre diminue l'absorption du cuivre. Aussi l'ingestion de sulfate de cuivre ne se répercute-t-elle qu'indirectement sur l'utilisation du métal, à cause des aléas du tractus intestinal. On ne connaît pas de système régulateur de l'absorption du cuivre. Néanmoins, d'après Van Campen [12] le mécanisme, quoique complexe, peut se résumer ainsi : si la concentration en cuivre dans le tractus gastro-intestinal est faible, 
l'absorption est plus forte que si elle était simplement proportionnelle à la concentration en métal. Mais lorsque le cuivre intestinal croît, l'absorption tend à devenir proportionnelle à la concentration. On peut conclure, à partir de ces faits expérimentaux, que le phénomène d'absorption met en œuvre au moins deux mécanismes qui agissent au niveau de la cellule intestinale.

\section{Transport}

Après son absorption intestinale, le cuivre est transporté par la voie sanguine.

Les trois " types de transporteurs" peuvent se ramener à :

- une métalloprotéine, la céruloplasmine, de poids moléculaire 151000 ,

- un complexe réversible entre le cuivre et les albumines sériques,

- le cuivre " échangeable " lié aux acides aminés.

D'après Underwood [13], le schéma qu'il convient de considérer comme le plus logique pour le transport du cuivre est le suivant : le cuivre alimentaire absorbé, se fixe sur les albumines du sang. Il est alors dirigé vers le foie où serait formée la céruloplasmine. Cette protéine constitue une forme stable de cuivre lié, et ne libère le cuivre qu'à l'échelon cellulaire, lorsqu'elle est renouvelée. Dans ces conditions la troisième forme, acides aminés-cuivre, forme échangeable et mobile du cuivre, serait en équilibre avec la forme albumine sérique, et faciliterait non seulement le transport mais encore la pénétration du cuivre au niveau cellulaire. Il s'ensuit que le cuivre, dans ses fractions circulantes reste toujours lié aux protéines sériques ou à leurs fractions constitutives. Nous avons pu observer [14] le même phénomène sur le lactosérum de lait de vache, le cuivre se répartissant sur les protéines ou sur les acides aminés.

\section{Stockage}

Le foie constitue le principal organe de stockage du cuivre. L'âge et l'alimentation de l'animal, ainsi que la gestation, peuvent faire varier la capacité du foie à stocker le cuivre. Le cuivre restant est réparti dans tous les tissus et dans les liquides circulants. La littérature donne des valeurs du cuivre hépatique allant de 10 à $50 \mathrm{mg}$ par kg de matière sèche (p.p.m.). D'ailleurs, il semble bien que ce soit la vache en début de gestation et le veau à la naissance qui présentent les accroissements les plus spectaculaires du taux hépatique normal, jusqu'à 400 p.p.m. selon Underwood [13]. De toute façon, la teneur sanguine, de l'ordre de 1 p.p.m., reste toujours nettement inférieure à la teneur hépatique. Plus récemment, Pennigton 
[15] cite pour le foie des chiffres moyens de 28 et 79 p.p.m. respectivement pour la vache et le veau, obtenus avec plus de 50 déterminations, mais rapportés au poids de l'organe frais.

\section{Excrétion}

La voie fécale est la voie préférentielle, qui assure à elle seule plus de 95 p. 100 de l'excrétion normale, dont les $4 / 5$ proviennent de la bile. Dans cette bile, le cuivre est généralement lié aux nombreuses molécules organiques que le foie élimine. Les urines n'éliminent que très peu cet élément.

\section{IV. - IMPORTANCE BIOCHIMIQUE ET BIOLOGIE DU CUIVRE}

Le cuivre dans les tissus animaux se présente sous trois formes chimiques qui sont toutes des cuproprotéines : la céruloplasmine, la cérébrocupréine et les enzymes à cuivre ou cupro-enzymes.

La céruloplasmine, de poids moléculaire 151000 , contient 8 atomes de cuivre par molécule. Le cuivre de cette protéine est incorporé au niveau des microsomes des cellules hépatiques, et ne peut plus être libéré in vivo. La cérébrocupnéine, que Carrico et Deutsh [16] ont récemment identifiée par des études physiques, chimiques et immunologiques à l'érythrocupréine et à l'hépatocupréine, a un poids moléculaire de l'ordre de 35000 . Elle contient 2 atomes de cuivre, et son rôle biochimique n'a pas encore été élucidé, quel que soit l'organe où elle a été trouvée.

Les cupro-enzymes sont nombreuses, puisque Vallée et Wacker [17] en dénombrent 19 dans les règnes animaux et végétaux. Dans le cas des bovins, il convient de signaler en premier lieu la cytochrome oxydase de cœur de bœuf, qui constitue le dernier maillon de la chaîne respiratoire. Il y a 1 atome de cuivre par hème, le hème constituant le co-facteur de la réaction enzymatique. Les valences du cuivre diffèrent, dans la cytochrome oxydase, du cytochrome a au cytochrome as. Ceci explique la fonction catalytique de la cytochrome oxydase, qui permet le passage des électrons depuis le cytochrome c réduit jusqu'à l'oxygène, et par là-même la respiration cellulaire.

- La tyrosinase (ou polyphényl-oxydase) dans tous les tissus des mammifères permet l'oxydation de la tyrosine en mélamine, le pigment noir de la peau, qui donne en particulier la coloration des poils, cornes, etc.

- Les amines oxydases, plasmatiques ou intramitochondriales, qui permettent la destruction de l'histamine, de la tryptamine et des catécholamines, jouant ainsi un rôle très important dans le métabolisme de l'animal. 
Le cuivre rentre aussi dans de nombreuses synthèses biochimiques dont le mécanisme n'est pas encore élucidé. Parmi celles-ci il faut citer la synthèse du protohème, de la kératine, et surtout des phospholipides myéliniques. Toutefois si les diverses fonctions du cuivre sont pressenties et évidentes, les modes d'action appartiennent toujours au domaine des hypothèses. Enfin le cuivre intervient directement dans la biochimie de nombreux autres éléments minéraux, par les interrelations métaboliques, comme le prouve le mécanisme des carences.

\section{v. - LeS VARIATIONS PATHOLOgIQUES OU ACCIDENTELLES DU CUIVRE CHEZ LA VACHE}

Elles traduisent deux types de réaction de l'animal à des conditions anormales : les carences ou les empoisonnements, qui sont dues à des variations des taux physiologiques, et les maladies à origine biochimique qui sont dues à un disfonctionnement métabolique interne.

Notre propos n'est pas ici de faire une revue de tous les cas possibles, car Bellanger J., Lamand M. et Perigaud S. [18] s'en sont déjà chargés dans une revue très complète. Néanmoins les cas de carence présentent pour nous un gros intérêt dans la mesure où ils peuvent influencer la composition du lait, et leur influence économique n'est pas du tout négligeable : ce sont les anémies nutritionnelles, dont la liste relevée par le Commonwealth Bureau of Animal Nutrition [3] dénonce l'existence un peu dans tous les pays du monde. La carence en cuivre chez les bovins peut avoir de nombreuses origines, dont les plus fréquentes sont liées à des facteurs agronomiques. Ces facteurs agronomiques peuvent d'ailleurs combiner leurs effets, ce qui accroît leur influence. En dehors de la composition même du sol, il y a la faculté d'assimilation du cuivre par les végétaux, leur teneur en "cuivre utilisable " pour l'animal, le climat qui peut varier d'une année sur l'autre en influençant la composition minérale, la fertilisation éventuelle du sol, et aussi les antagonismes entre oligo-éléments (cuivre-molybdène par exemple). La carence se traduit chez les bovins par une décoloration des poils, des troubles osseux plus ou moins discrets, et par une grosse perte à la production (croissance, lactation et reproduction). Analytiquement, cette carence se traduit par un seuil critique du cuivre hépatique de $25 \mathrm{mg}$ par $\mathrm{kg}$ de matière sèche, quoique ce chiffre soit contesté. En zootechnie, on a défini de nombreux essais de correction des carences lorsqu'elles sont pressenties à temps.

L'autre danger est celui de la toxicité. Parfois cette toxicité du cuivre doit être considérée comme le résultat d'un essai de correction de la carence. Ainsi chez les bovins peut-on administrer " per os " jusqu'à $2 \mathrm{~g}$ de $\mathrm{CuSO}_{4}$ par jour pendant un temps variant de 
5 à 18 semaines, mais il existe pour les aliments une dose toxique de l'ordre de $200 \mathrm{mg}$ par $\mathrm{kg}$ pour Cunningham cf. Jacquot [9]. En fait, un animal préalablement carencé est beaucoup plus sensible à la toxicité du cuivre. Ammerman [19] présente la toxicité sous deux formes. La première phase, qui ne présente aucun des symptômes usuels, consiste en une phase d'accumulation passive dans les tissus (et surtout dans le foie). La deuxième phase est caractéristique, c'est l' " anémie hémolytique ». Cette crise hémolytique se traduit par des concentrations maximales de cuivre dans le foie et dans le sang, et elle peut être suivie de la mort de l'animal. Comme les carences sont les cas pathologiques les plus fréquents, les traitements de médecine vétérinaire des cas pathologiques consistent surtout en un apport de cuivre. Il y a possibilité d'un apport minéral alimentaire, par incorporation, ou à l'aide de la " pierre à cuivre ". Dans ce dernier cas, Bellanger, Lamand et Périgaud [18] signalent que les consommations peuvent varier dans un rapport de 1 à 100 en fonction des animaux. Aussi préfère-t-on l'injection d'un sel de cuivre qui possède une toxicité réduite, une bonne absorption et qui n'entraine chez l'animal que des lésions minima. C'est le glycinate de cuivre qui recueille les vœux de tous les auteurs, quoique la fréquence et la concentration des injections n'aient pas encore été bien définies. Dans tous les cas, on s'est intéressé au devenir de ces additions artificielles de cuivre à cause d'une éventuelle variation de la teneur en cuivre du lait, teneur qui influence sa sensibilité à l'oxydation. Dunkley [20] a montré que, à part pendant les premières $24 \mathrm{~h}$, le taux de cuivre dans le lait reste constant après l'injection. Underwood [13] a prouvé que l'accroissement du cuivre alimentaire n'augmentait pas la teneur en cuivre du lait.

Mulder [21], après une longue discussion, arrive aussi à ce résultat.

D'autre part il semble bien que, en cas d'augmentation de la teneur en cuivre du lait par un traitement quelconque, le rôle catalytique de l'élément ne soit pas accru vis-à-vis de l'oxydation. On peut expliquer ce fait par un changement de réactivité du cuivre, ce cuivre " ajouté » étant devenu « passif ». Toutefois il convient de ne pas oublier que certaines contaminations du lait par le cuivre peuvent être causées plus simplement et sans l'intermédiaire du filtre biochimique que constitue l'animal.

Ammerman [19] cite aussi un cas curieux où l'on a obtenu une contamination grave du lait par le cuivre : la prairie avait été traitée au $\mathrm{C}_{\mathrm{T}} \mathrm{SO}_{4}$ et les mamelles étaient les principaux agents de contamination par simple frottement sur l'herbe...

Enfin, il convient de citer les divers résidus phytosanitaires qui peuvent amener, sous formes de poudres ou de pulvérisations, des quantités non négligeables de sels de cuivre (sulfate, arseniate, carbo- 
nate, naphthenate, etc.) ou d'oxydes. Ces résidus peuvent accidentellement atteindre la nourriture du bétail, et entraîner une toxicité passagère.

De plus, en voulant parfois amender des sols où les végétaux étaient déjà carencés, on est arrivé à rompre définitivement un équilibre biologique précaire ce qui a pour conséquence immédiate d'accroître la carence.

\section{VI. - CONCLUSION}

L'équilibre nutritionnel de la vache quant à l'élément cuivre pose un problème complexe dont la solution doit avant tout tenir compte de tous les paramètres locaux et de leur importance relative. D'autant plus que, trop souvent, les résultats des recherches effectuées s'appliquent à des races bien définies, mais différentes, et leur rapprochement systématique omet le facteur " génétique " dont l'influence n'est encore que pressentie.

Toutefois, il résulte de cette revue que la vache, dans des conditions normales de nourriture, ne présente pratiquement jamais de troubles liés à la toxicité du cuivre, puisque Cunnigham cite même [22] des absorptions journalières de 0,8 à $5 \mathrm{~g}$ de sulfate de cuivre pendant 9 mois sans symptôme toxique, chez le jeune veau comme chez la vache adulte. Au contraire, le rôle biologique important du cuivre étant indiscutable, surtout chez le jeune en croissance, et les cas de carence paraissant relativement nombreux, il semble judicieux de conseiller un apport alimentaire important de cuivre chez les bovins, sous une forme naturelle dans la nourriture. En effet, une nutrition mieux équilibrée ne pourrait qu'améliorer le " rendement » global du bétail, donc les intérêts des fermiers et éleveurs. De plus ce traitement n'intervenant pas défavorablement sur la technologie laitière (le cuivre ne passe pas dans le lait), pourquoi donc ne pas le pratiquer systématiquement ? La question est posée, et le débat ouvert, ce qui constitue le but de notre propos.

\section{S u m m a r y}

In this review is presented the problem of copper in the feed of dairy cows. The author describes briefly feed sources and contamination risks, gives an evaluation of nutrional need and a view of metabolism of copper in the animal. Biological and biochemical importance is pointed out, also pathological and accidental variations. These nutritional facts encouraged the author to recommand a greatest copper intake of dairy cattle for a better productivity of the animals. 


\section{Bibliographie}

[1] Dawis (W. L.) (1931). - Observations on the copper and iron content of milk and others dairy products. $J$. of Dairy Research, 3, 86.

[2] Randoin (L.) (1937). - Tables de composition des aliments. Hermann, Paris.

[3] Commonwealth Bureau of Animal Nutrition (1956). - Minerals in pastures; deficiency and excess in relation to animal health, 119 à 132.

[4] Jaulmes (P.) et Hamelle (G.) (1971). - Oligo-éléments dans les aliments. Annales de la nutrition et de l'alimentation, B 133 à B 203.

[5] Heckman (Mary) (1968). - Collaborative study of minerals in feeds. Journal of the A.O.A.C., vol. $51, \mathrm{n}^{\circ} 4,776$ à 779 .

[6] Agricultural Research Council (1965). - The nutrient requirements of farm livestock, $\mathrm{n}^{\circ} 2$ Cattle, 2, Londres.

[7] Golse (J.) (1933). - Recherche et dosage du cuivre dans les eaux. Etude de l'action des eaux sur les conduites en cuivre. Bull. Trav. Société de Pharmacie de Bordeaux, 1, 30.

[8] ILARI (J. L.). - Résultats non publiés.

[9] Jacouot et coll. (1964). - Nouvelle Encyclopédie Agricole. Vol. 3 : Nutrition Animale, 2086-2103, J. B. Baillière édit., Paris.

[10] Agricultural Research Council (1965). - The Nutrient Requirements of farm Livestock, $\mathrm{n}^{\circ} 2$ Cattle, 9, Londres.

[11] Comar (C. L.), Davis (G.), Singer (L.) (1948). - The fate of radioactive copper administered to the bovine. J. Biological Chem., 905-915.

[12] VAN CAMPEN (D. R.) (1971). - Absorption of copper from the gastrointestinal tract, 211-227, in Intestinal absorption of metal ions, by SKoRYNA and WALDRON-EDWARD, Pergamon press.

[13] UNDERWOOD (E. J.) (1962). - Copper, 48-99, in : Trace element in human and animal Nutrition, $2^{2}$ édition. Academic Press, New-York.

[14] IlaRi (J. L.) (1973). - Thèse : Analyse du cuivre dans les produits laitiers. U.E.R. de Nutrition. Université de Dijon.

[15] Pennigton (J. T.) and al. (1973). - Copper content of foods. J. of the Amer. Dietetic Association, vol. 63, $\mathrm{n}^{\circ}$ 2, août, 143-153.

[16] CARRico (R. J.) and Deutsch (H. F.) (1969). - Isolation of human hepatocuprein and cerebrocuprein. J. of Biol. Chem., vol. 244, $\mathrm{n}^{\circ}$ 22, 6087-6093.

[17] VALLÉE (B. L.) and WACKER (E. C.) (1970). - The Proteins, vol. 5 : Metalloproteins, $2^{\mathrm{e}}$ édition, Academic Press.

[18] Bellanger (J.), Lamand (M.) et Périgaud (S.) (1970). - La carence en cuivre chez les ruminants. Annales de la Nutrition et de l'Alimentation, 24, 1-62.

[19] Ammermann (C. B.) (1970). - Recent Developments in Cobalt and Copper nutrition. J. Dairy Science, vol. 53, $\mathrm{n}^{\circ}$ 8, 1097-1107.

[20] Dunkley (W. L.) and al. (1968). - Influence of dietary copper and ethylenediaminetetra-acetate on copper concentration and oxydative stability of Milk. J. Dairy Science, vol. 51, $\mathrm{n}^{\circ}$ 6, 863-866.

[21] MuldeR (H.) and at. (1964). - The copper content of cows' milk. Neth. Milk and Dairy Journal, 18, 52-65.

[22] Cunningham (I. J.) (1946). - New-Zealand Journal of Science and Techno$\log y, 27,372-381$. 